\title{
Tors et roches moutonnées en Laponie suédoise : antagonisme ou filiation?
}

\section{Tor-like Features and Roches Moutonnées in Swedish Lappland : Antagonistic or Affiliated Landforms? \\ Felsburgen und Rundhöcker im schwedischen Lappland : einander widersprechende oder verwandte Landformen?}

\author{
Marie-Françoise André
}

Volume 55, numéro 3, 2001

URI : https://id.erudit.org/iderudit/006852ar

DOI : https://doi.org/10.7202/006852ar

Aller au sommaire du numéro

Éditeur(s)

Les Presses de l'Université de Montréal

ISSN

0705-7199 (imprimé)

1492-143X (numérique)

Découvrir la revue

Citer cet article

André, M.-F. (2001). Tors et roches moutonnées en Laponie suédoise :

antagonisme ou filiation? Géographie physique et Quaternaire, 55(3), 229-242. https://doi.org/10.7202/006852ar

\section{Résumé de l'article}

Dans les socles des hautes latitudes, tors et roches moutonnées ont été utilisés pour découper l'espace englacé au Quaternaire en secteurs respectivement préservés et remodelés par les inlandsis. Sans mettre en cause la validité d'un tel « zonage " à l'échelle macrorégionale, l'étude du plateau d'Aurivaara (Laponie suédoise, $68^{\circ} \mathrm{N}$ ), accidenté de quinze tors inégalement remodelés par l'inlandsis scandinave, jette une nouvelle lumière sur les relations pouvant unir ces formes a priori antinomiques. Les résultats tirés de l'analyse des modelés de déchaussement et des dépôts glaciaires (morphométrie, pétrographie, granulométrie, état d'altération...) sont confrontés avec les observations effectuées sur les roches moutonnées voisines de Laponie norvégienne, récurées par les glaces tardi-weichseliennes. Quatre conclusions se dégagent : 1 ) les tors d'Aurivaara sont très probablement des formes pré-quaternaires ; 2) la tendance lourde à un régime à base froide est attestée par la prépondérance de l'accumulation sur l'ablation, sous la forme du " parachutage » répété de matériel morainique, confirmant la thèse du " palimpseste glaciaire » ; 3) les tors constituent de précieux indicateurs de la tranche d'ablation glaciaire quaternaire dont la minceur, en situation d'interfluve (0-12 m), contraste avec l'ampleur du surcreusement (proche de $200 \mathrm{~m}$ ) de l'ombilic du lac Torneträsk ; 4) l'existence d'un continuum menant du tor à la roche moutonnée reste à expliquer en ce qui a trait à la dynamique glaciaire. 


\section{TORS ET ROCHES MOUTONNÉES EN LAPONIE SUÉDOISE : ANTAGONISME OU FILIATION?}

Marie-Françoise ANDRÉ, Laboratoire de Géographie physique (UMR 6042-CNRS), Université Blaise-Pascal, Maison de la Recherche, 4, rue Ledru, 63057 Clermont-Ferrand, Cedex 1, France.

RÉSUMÉ Dans les socles des hautes latitudes, tors et roches moutonnées ont été utilisés pour découper l'espace englacé au Quaternaire en secteurs respectivement préservés et remodelés par les inlandsis. Sans mettre en cause la validité d'un tel «zonage» à l'échelle macrorégionale, l'étude du plateau d'Aurivaara (Laponie suédoise, $68^{\circ} \mathrm{N}$ ), accidenté de quinze tors inégalement remodelés par l'inlandsis scandinave, jette une nouvelle lumière sur les relations pouvant unir ces formes a priori antinomiques. Les résultats tirés de l'analyse des modelés de déchaussement et des dépôts glaciaires (morphométrie, pétrographie, granulométrie, état d'altération...) sont confrontés avec les observations effectuées sur les roches moutonnées voisines de Laponie norvégienne, récurées par les glaces tardi-weichseliennes. Quatre conclusions se dégagent : 1) les tors d'Aurivaara sont très probablement des formes pré-quaternaires; 2) la tendance lourde à un régime à base froide est attestée par la prépondérance de l'accumulation sur l'ablation, sous la forme du «parachutage » répété de matériel morainique, confirmant la thèse $\mathrm{du}$ «palimpseste glaciaire "; 3 ) les tors constituent de précieux indicateurs de la tranche d'ablation glaciaire quaternaire dont la minceur, en situation d'interfluve (0-12 m), contraste avec l'ampleur du surcreusement (proche de $200 \mathrm{~m}$ ) de l'ombilic du lac Torneträsk ; 4) l'existence d'un continuum menant du tor à la roche moutonnée reste à expliquer en ce qui a trait à la dynamique glaciaire.
ABSTRACT Tor-like features and roches moutonnées in Swedish Lappland : antagonistic or affiliated landforms? In previously glaciated nordic shield areas, roches moutonnées and tor-like features were fruitfully used at a macroregional scale to separate scoured and nonscoured zones. However, such a dichotomic view has to be modified when working at a local scale where relationships between tors and roches moutonnées appear more complex. It is the case on the Aurivaara Plateau (Swedish Lapland, $68^{\circ} \mathrm{N}$ ) where are found fifteen tor-like features that have been unevenly modified by the Scandinavian Ice Sheet. The results of detailed analyses of smallscale landforms and glacial deposits (morphometry, lithology, grain size, weathering stages, etc.) are compared with previous observations made on the nearby Late Weichselian roches moutonnées from Norwegian Lapland. The conclusions are fourfold: 1 ) the Aurivaara tor-like features are most probably pre-Quaternary landforms; 2) a dominantly cold-based glacial regime is suggested based on the preponderance of accumulation over ablation (deposition of morainic material at the end of each glaciation), which is in accordance with the idea of "a glacial palimpsest"; 3) tor-like features are valuable indicators to quantify the overall Quaternary glacial erosion that is weak on tabular interfluves such as the Aurivaara Plateau (0-12 m), in contrast with the $200 \mathrm{~m}$ overdeepening of the nearby Torneträsk basin; 4) the existence of a continuum leading from the well-preserved tors to the ice-profiled roches moutonnées remains partly unexplained from a dynamical point of view.
ZUSAMMENFASSUNG Felsburgen und Rundhöcker im schwedischen Lappland : einander widersprechende oder verwandte Landformen? Im vormals mit Inlandeis vergletscherten skandinavischen Schild wurden Rundhöcker und Felsburgen dazu benutzt, Gebiete mit und ohne Glazialerosion im regionalen Maßstab voneinander abzugrenzen. Im kleinräumigen Bereich muß dieser dichotomische Ansatz allerdings modifiziert werden, da die Verhältnisse zwischen den Rundhöckern und den Felsburgen weit komplizierter und komplexer zu sein scheinen. So beispielsweise im Falle des Aurivaara-Plateaus

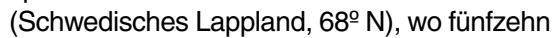
Felsburgen-ähnliche und durch das Inlandeis in sehr unterschiedlicher Weise überformte Formen untersucht wurden. Die eingehende Analyse der kleinräumigen Formen und der Gletscherablagerungen mit Hilfe morphometrischer, petrographischer, korngrössenanalytischer Untersuchungen sowie anhand des Verwitterungsgrades und weiterer Verfahren werden mit früheren Beobachtungen auf den nahe gelegenen Rundhöckern des SpätWeichsels im norwegischen Lappland verglichen. Schlußfolgernd gilt : 1 . Die Felsburgenähnlichen Formen des Aurivaara-Plateaus sind wahrscheinlich prä-quartären Alters; 2. Aufgrund der Dominanz der Akkumulation gegenüber der Ablation (das Moränenmaterial wurde jeweils am Ende des Glazials abgelagert) wird im Einklang mit der Vorstellung des "Glazialen Palimpsests" gefolgert, dass die Unterseite des Eises kalt und spröde war; 3. Felsburgen-ähnliche Formen ermöglichen die Abschätzung der glazialen Erosion mit geringen Werten im Bereich flacher Wasserscheiden (z.B. im Fall des Aurivaara-Plateaus, 0-12 m) oder im Gegensatz dazu mit einer Übertiefung von $200 \mathrm{~m}$ im nahe gelegenen Becken von Torneträsk; 4. Graduelle Übergänge von guterhaltenen Felsburgen zu gletschergeformten Rundhöckern bleiben aus dynamischer Sicht teils ungeklärt. 


\section{INTRODUCTION}

Dans les socles des hautes latitudes englacés au Quaternaire froid, roches moutonnées et «tors " ${ }^{1}$ sont généralement considérés comme des formes emblématiques, indicatrices de contextes glaciologiques très contrastés. Les premières figurent, en effet, au premier rang des formes classiques d'érosion glaciaire puisqu'elles sont le produit de l'abrasion et du délogement opérés par un glacier mobile à base chaude (Sugden et John, 1976; Bennett et Glasser, 1997). À l'inverse, les tors sont considérés comme des modelés d'altération hérités, dont la conservation s'explique par leur recouvrement sous une chape immobile de glace à base froide (Sugden et Watts, 1977; Söderman et al., 1983; Jonsson, 1983; Watts, 1983; Kaitanen, 1989; Kleman et Hättestrand, 1999).

C'est en s'appuyant très largement sur la distribution spatiale des tors et des roches moutonnées que Sugden (1974), Dyke (1993) et Kleman (1994) ont découpé l'espace englacé au Quaternaire en zones décapées et non décapées par la glace, dessinant les contours d'une véritable géographie de l'érosion glaciaire associée à une logique générale de répartition des régimes thermiques des inlandsis groenlandais, scandinave et nord-américain. Mais si le zonage ainsi proposé emporte souvent l'adhésion à l'échelle macrorégionale, il en va différemment à l'échelle locale. Sur le terrain, il arrive, en effet, que la dichotomie tors/roches moutonnées s'efface pour laisser place à un gradient suggérant un continuum dynamique menant du tor à la roche moutonnée. C'est du moins ce que laissent à penser certaines observations antérieurement effectuées en Terre de Baffin, au Labrador et en Laponie finlandaise (Sugden et Watts, 1977 ; Gangloff, 1983; Kaitanen, 1989). Et c'est ce que vient établir avec netteté, nous semble-t-il, la présente étude du plateau d'Aurivaara (Laponie suédoise), riche d'une quinzaine de tors inégalement remodelés par la glace. Sur la base de l'étude croisée des modelés de détail et du matériel autochtone et allochtone, l'analyse de ce site apporte des éléments nouveaux concernant : 1) les modalités spatio-temporelles d'action de l'inlandsis scandinave au long du Quaternaire;2) l'évaluation de son impact géomorphologique dans le cadre d'une approche à la fois qualitative (typologie des formes fondée sur le degré d'efficacité érosive de la glace) et quantitative (essai d'évaluation d'une tranche d'ablation glaciaire représentative des secteurs d'interfluves tabulaires).

\section{TERRAIN D'ÉTUDE}

Situé à $68^{\circ} \mathrm{N}$, à $30 \mathrm{~km}$ au nord-ouest de Kiruna et à $15 \mathrm{~km}$ au sud du lac Torneträsk, le plateau d'Aurivaara culmine à $850 \mathrm{~m}$ d'altitude et domine de plus de $300 \mathrm{~m}$ les vallées environnantes (fig. 1). Ce plateau appartient au bouclier fennoscandien, d'âge précambrien, qui affleure à l'est du front de chevauchement des Calédonides. Une quinzaine de modelés de déchaussement de type tor s'y trouvent disséminés sur quelque

1. Rappelons que le terme «tor » désigne un «volume rocheux résiduel, enraciné, d'échelle décamétrique, constitué par un empilement géométrique de blocs aux arêtes émoussées, et provenant principalement du dégagement partiel des produits meubles d'une cryptodécomposition différentielle » (Godard, 1966, p. 169).

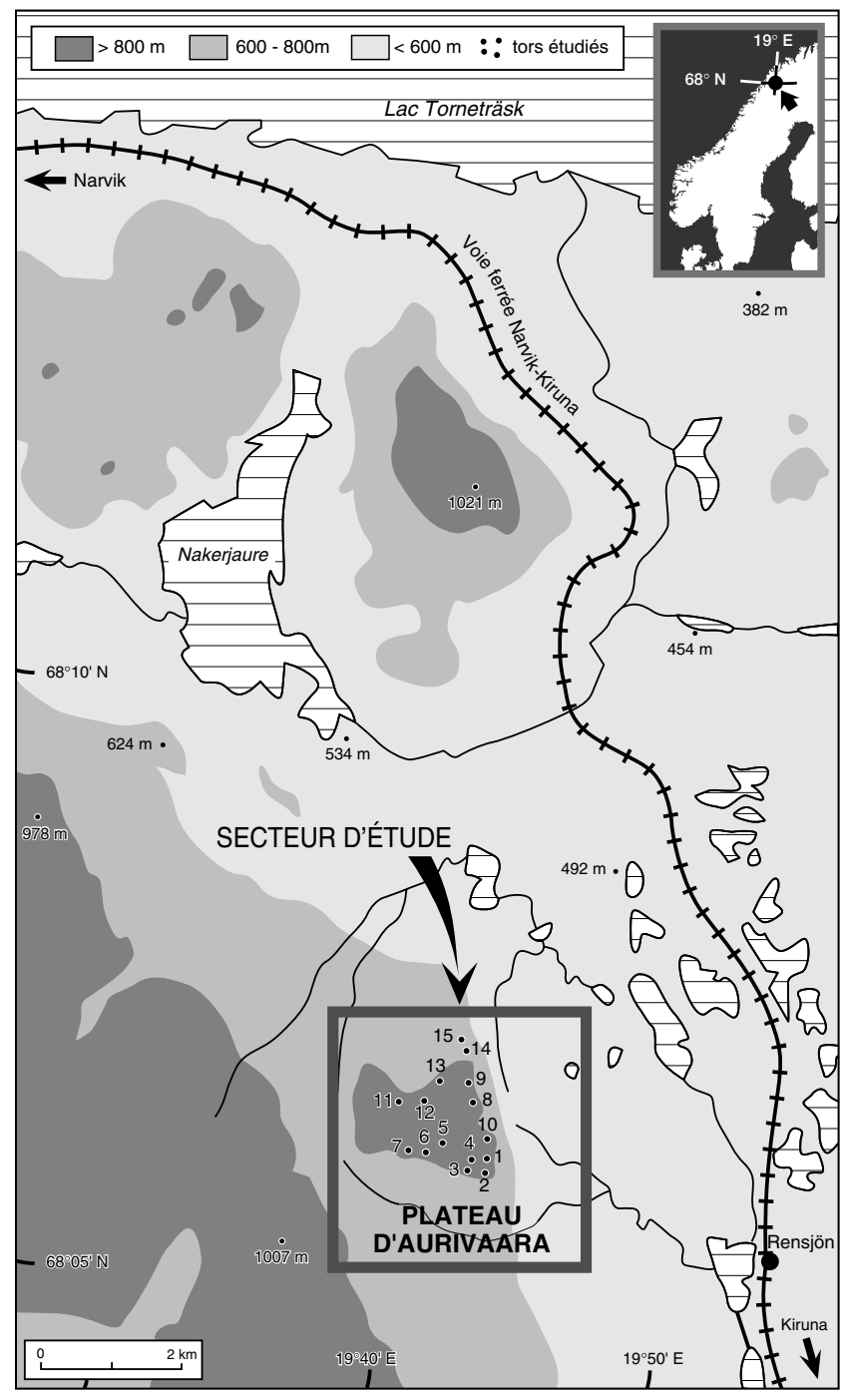

FIGURE 1. Localisation du site d'étude (plateau d'Aurivaara, Laponie suédoise, $68^{\circ} \mathrm{N}$ ). Les tors sont numérotés de 1 à 15.

Location of the study area (Aurivaara Plateau, Swedish Lappland, $\left.68^{\circ} \mathrm{N}\right)$. The tor-like features are numbered from 1 to 15.

$8 \mathrm{~km}^{2}$, dont trois au cœur du plateau (2-6 m de haut) et douze en périphérie, dont certains atteignent la hauteur maximale de 10 à $12 \mathrm{~m}$. Vers l'intérieur du plateau, ces tors dominent fréquemment, à la faveur d'un knick, des topographies planes très faiblement inclinées (de 1 à $4^{\circ}$ ) au profil tendu, évoquant des éléments de pédiments. Ceux-ci prennent de l'ampleur au cœur du plateau où ils ne sont accidentés que par quelques rarissimes îlots rocheux aux formes surbaissées, là où ils ne laissent pas place à une topographie mollement vallonnée.

Tous les tors sont taillés dans des affleurements de syénite gris sombre, caractérisés par leur massiveté (espacement des diaclases jusqu'à 3,70 m), leur grain grossier et leur sensibilité à la météorisation sphéroïdale (fig. 2). Ce faciès contraste avec les rares venues de syénite rose, à débit anguleux (espacement des diaclases $=20 \mathrm{~cm}$ ), que jalonnent de minces champs de blocaille. Quant à la composition minéralogique des syénites d'Aurivaara, elle est naturellement dominée par 
FIGURE 2. Altération en boules des syénites d'Aurivaara (tor $\left.\mathrm{n}^{\circ} 11\right)$. Cliché M.-F. A.

Spheroidal weathering of the Aurivaara syenites (tor No. 11).

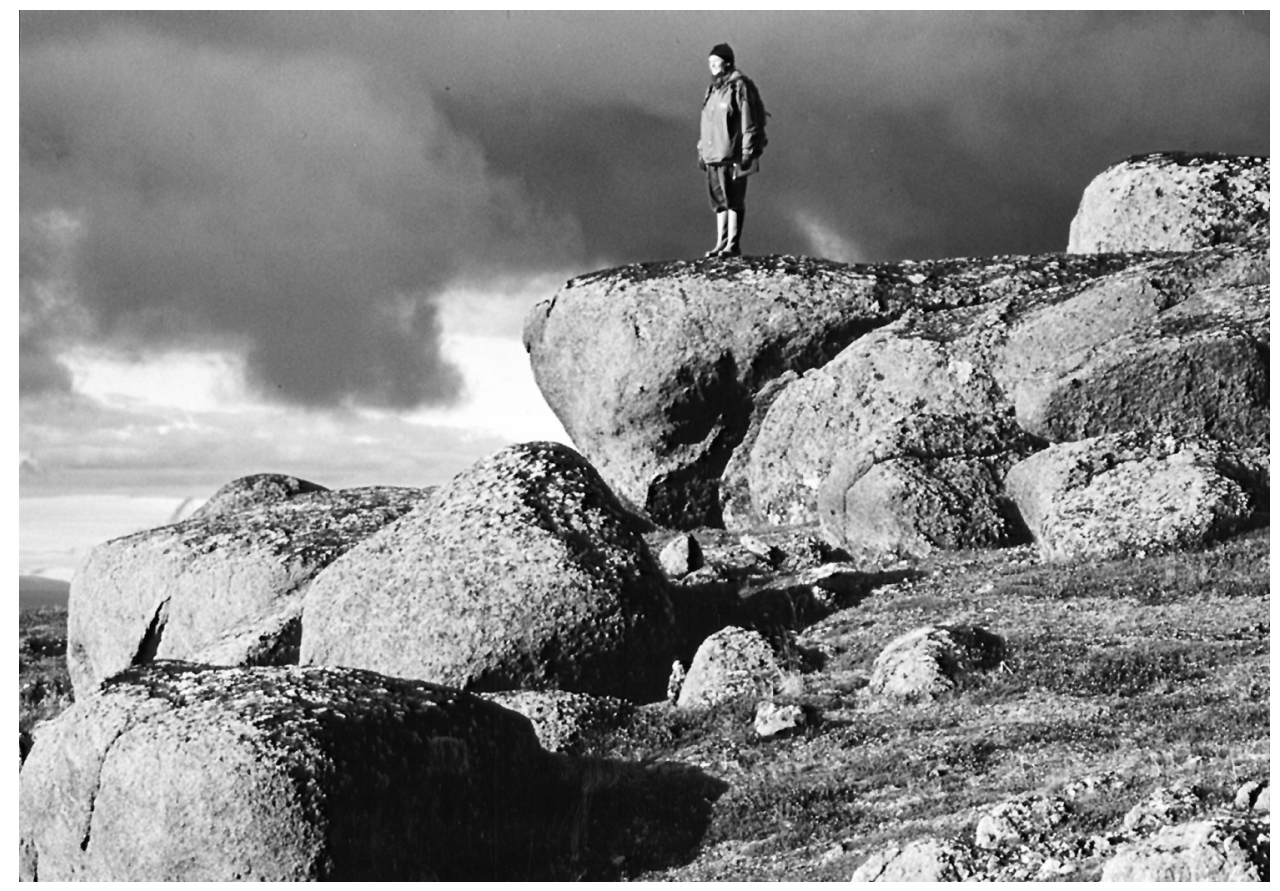

les feldspaths. II s'agit pour l'essentiel de microclines centimétriques mais en lumière polarisée, la trame moirée des macles en tartan laisse deviner par endroits quelques macles de Carlsbad résiduelles caractéristiques des orthoses. Les feldspaths sont accompagnés de ferro-magnésiens, essentiellement des pyroxènes (augite et diopside) et des opaques (magnétite), plus rarement des amphiboles (hornblende) et des biotites. Certaines des lames minces réalisées au Laboratoire de Géologie de l'Université de Limoges sont riches en apatite et renferment des minéraux accessoires comme le zircon. Le quartz est absent ou rarissime.

Les témoignages abondent qui attestent du recouvrement du plateau d'Aurivaara par l'Inlandsis scandinave. II s'agit essentiellement de dépôts glaciaires, et notamment d'un revêtement morainique, mince (10 cm en moyenne) mais généralisé, qui s'épaissit dans les dépressions situées entre les tors (jusqu'à $1 \mathrm{~m}$ ) où la matrice morainique est visible au cœur de sols structurés (ostioles et ébauches de cercles de pierres). Ailleurs, le vannage éolien ne laisse subsister en surface qu'un pavage grossier d'une grande hétérogénéité pétrographique. La partie la plus déprimée du plateau, qui recèle une mare partiellement asséchée, montre un épandage fluvioglaciaire de deux mètres d'épaisseur, dont les galets sont parfaitement roulés (fig. 3). Mais le témoignage de l'englacement le plus frappant dans le paysage est sans nul doute le semis de blocs erratiques (jusqu'à $2 \mathrm{~m}$ de long) qui jalonnent le sommet de tous les tors et sont piégés jusqu'à 2,20 $\mathrm{m}$ de profondeur dans les diaclases verticales (fig. 4), et jusqu'à 3,40 m dans les diaclases obliques.

\section{MÉTHODES}

À partir d'un camp de base situé au cœur du plateau d'Aurivaara, chacun des quinze tors a fait l'objet d'investigations systématiques. Des données ont été collectées concer- nant la géométrie des modelés de déchaussement (dimensions, angulosité, etc.), la présence à leur surface d'arènes in situ, de microformes d'altération (vasques, « rochers champignons », pierres branlantes en cours d'arénisation, écailles de desquamation) et de témoignages éventuels d'un remodelage glaciaire. La largeur et la profondeur des diaclases séparant les boules rocheuses constitutives des tors ont été évaluées sur la base de 140 mesures par tor. La profondeur maximale de pénétration du matériel morainique piégé par les diaclases a été relevée dans chaque site où a été également dressé le spectre pétrographique des blocs erratiques disséminés en surface (50 blocs par tor). L'état d'altération de ces derniers a été apprécié à partir de critères de terrain (résistance au marteau, présence et épaisseur des cortex, etc.). L'épaisseur des revêtements morainiques et des placages fluvioglaciaires a été également relevée, de même que les indications sur le sens d'écoulement glaciaire fournies par les stries glaciaires et les boules rocheuses sub-in situ transportées à contrepente sur de très faibles distances.

Les 68 échantillons meubles et rocheux prélevés à Aurivaara ont été traités en laboratoire. Les échantillons meubles ont fait l'objet d'observations morphoscopiques et d'analyses granulométriques sur granulomètre laser Coulter LS 230 visant notamment à distinguer des générations de dépôts et à mettre en évidence des mélanges d'apports. L'état d'altération des affleurements rocheux a été apprécié sur la base de l'examen de lames minces qui ont été confrontées avec profit à celles réalisées sur 13 échantillons provenant des roches moutonnées tardi-weichseliennes fermant à l'amont le bassin du lac Torneträsk. Plus généralement, une comparaison systématique a été conduite entre les tors d'Aurivaara et les roches moutonnées de Riksgränsen (André, 1995) pour mieux évaluer le degré d'évolution des affleurements en l'absence de coupes dans les arènes sur le site d'étude. 
FIGURE 3. Épandage fluvioglaciaire colmatant une dépression au cœur du plateau d'Aurivaara. Cliché M.-F. A.

Glacifluvial deposit in a hollow in the heart of the Aurivaara plateau.

FIGURE 4. Erratique de granite rose piégé à l'intérieur d'une diaclase dans les syénites gris sombre (tor $n^{\circ} 5$ ). Cliché M.-F. A.

Pink granite erratic trapped inside a joint in dark grey syenite (tor No. 5).
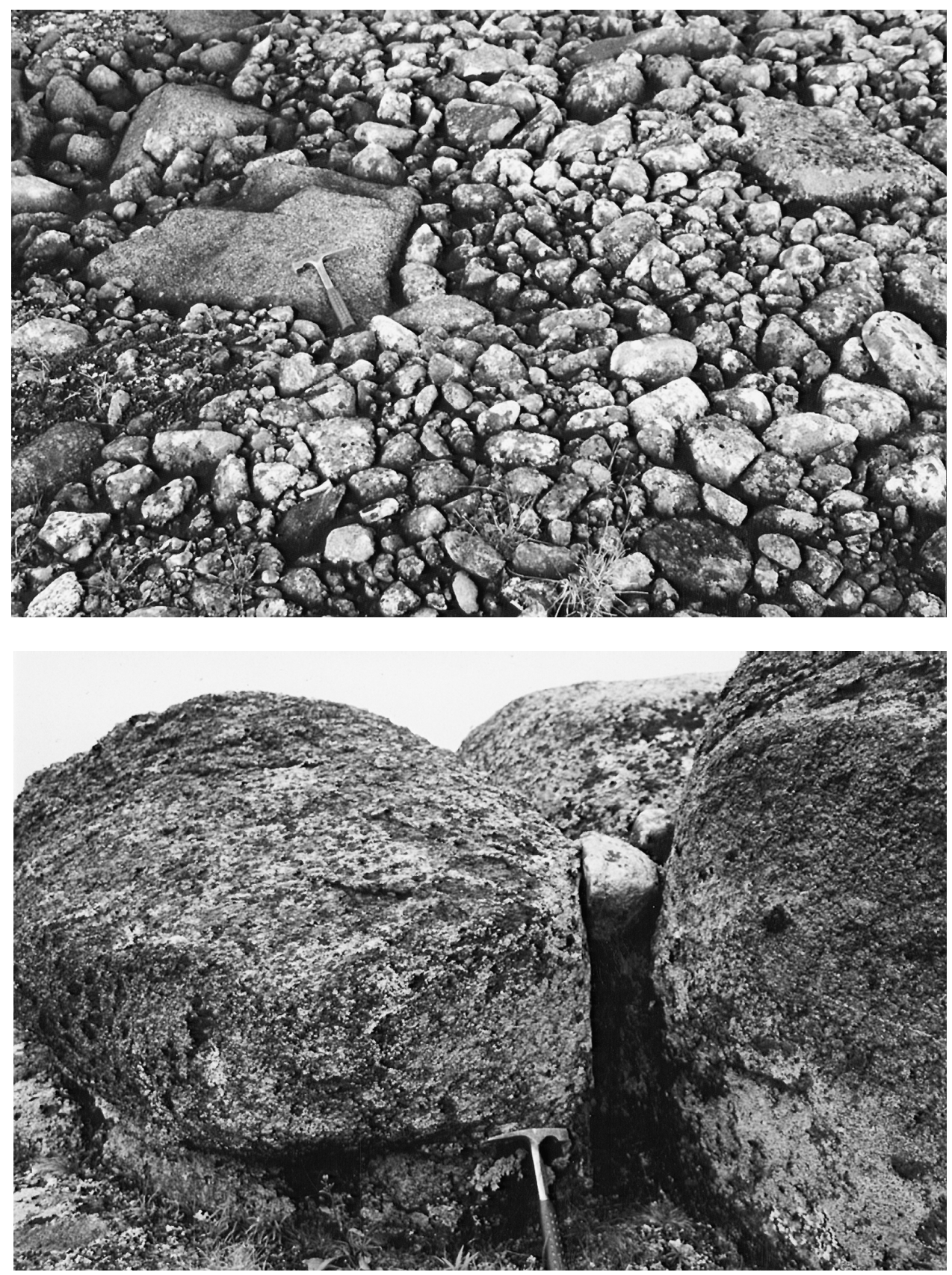

\section{PÉTROGRAPHIE DES ERRATIQUES ET SENS D'ÉCOULEMENT GLACIAIRE}

Les spectres pétrographiques élaborés sur la base de l'examen de 50 erratiques par tor font apparaître une forte dominante $(70 \%)$ de roches granito-gneissiques dont les trois quarts de granite rose, fréquemment mylonitisé. En moindre proportion sont représentés les quartzites s.l. $(22 \%)$ et les amphibolites (5\%) auxquels s'ajoutent quelques rares dolérites (tabl. I et fig. 5). On soulignera que la dénomination "quartzite » recouvre ici divers faciès dont d'authentiques quartzophyllades. D'une manière générale, les quartzites sont surreprésentés dans la mesure où il est apparu, à l'examen des lames minces, que certaines mylonites avaient été identifiées à tort par l'auteur, sur le terrain, comme des quartzites.

Au total, l'écrasante majorité d'éléments granito-gneissiques issus du Bouclier fenno-scandien reflète un écoulement principal de l'Inlandsis scandinave en direction de l'ouest, la ligne de partage des glaces se situant dans le prolongement du golfe de Botnie au maximum weichselien (Kleman, 1990). La présence plus ponctuelle d'amphibolites à grenat et surtout de quartzophyllades (hard schist) issues des Calédonides indique toutefois une provenance occidentale, sans doute à mettre en relation avec le déplacement, au cours du Quaternaire, de la ligne de partage des glaces. Une position plus occidentale, en direction des monts Kebnekaise, est d'ailleurs matérialisée par 

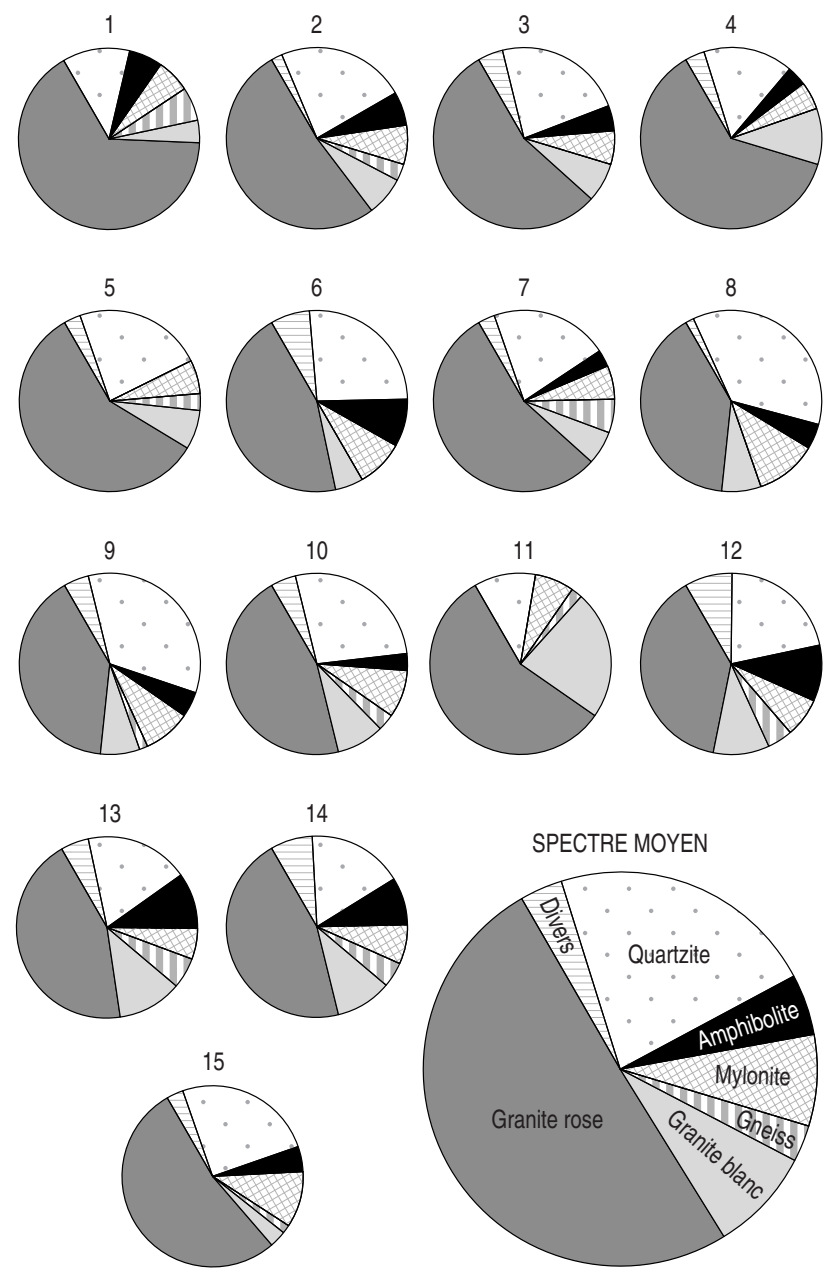

FIGURE 5. Spectres pétrographiques du semis d'erratiques coiffant les tors d'Aurivaara.

Lithological composition of the erratics scattered on top of tors at Aurivaara.

l'inclinaison vers l'est des chenaux juxtaglaciaires qui ceinturent le bassin du lac Torneträsk et ont été préservés sous un glacier à base froide (Kleman, 1992).

\section{TYPOLOGIE DES FORMES DE DÉCHAUSSEMENT}

Alors que les dépôts glaciaires sont omniprésents à Aurivaara, les formes d'érosion glaciaire apparaissent rarissimes comme le montre l'étude fine des modelés de déchaussement. Par delà une étonnante diversité morphologique, les 15 tors d'Aurivaara, dont les principales caractéristiques sont présentées sur le tableau II, peuvent être regroupés en trois grandes familles de formes, schématisées sur la figure 6 : les tors ayant conservé tout un cortège de micromodelés d'altération sommitaux (type 6A); les tors se limitant à un empilement de «sacs de laine », terme désignant des blocs plurimétriques de granite in situ, de forme parallélépipédique et aux angles émoussés (type 6B); les tors réduits à l'état de «racines 》 polies, striées, voire profilées par la glace (type 6C).

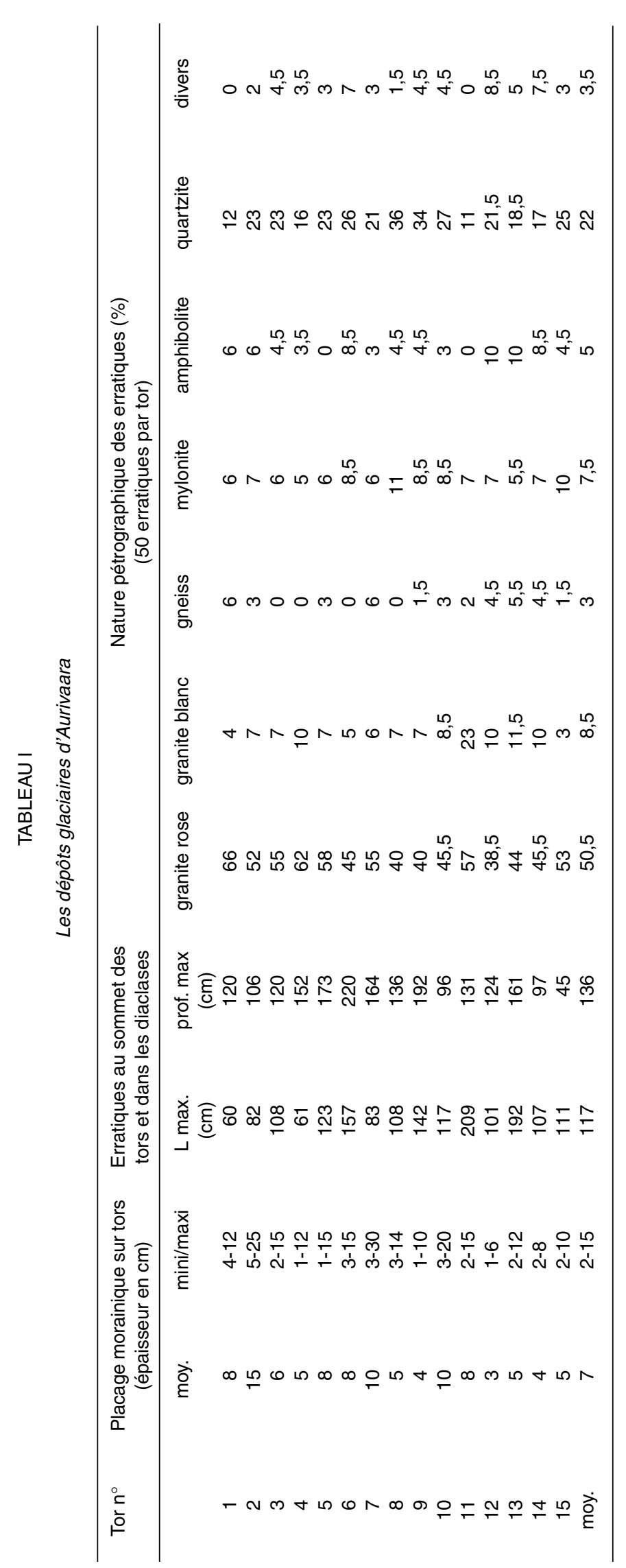



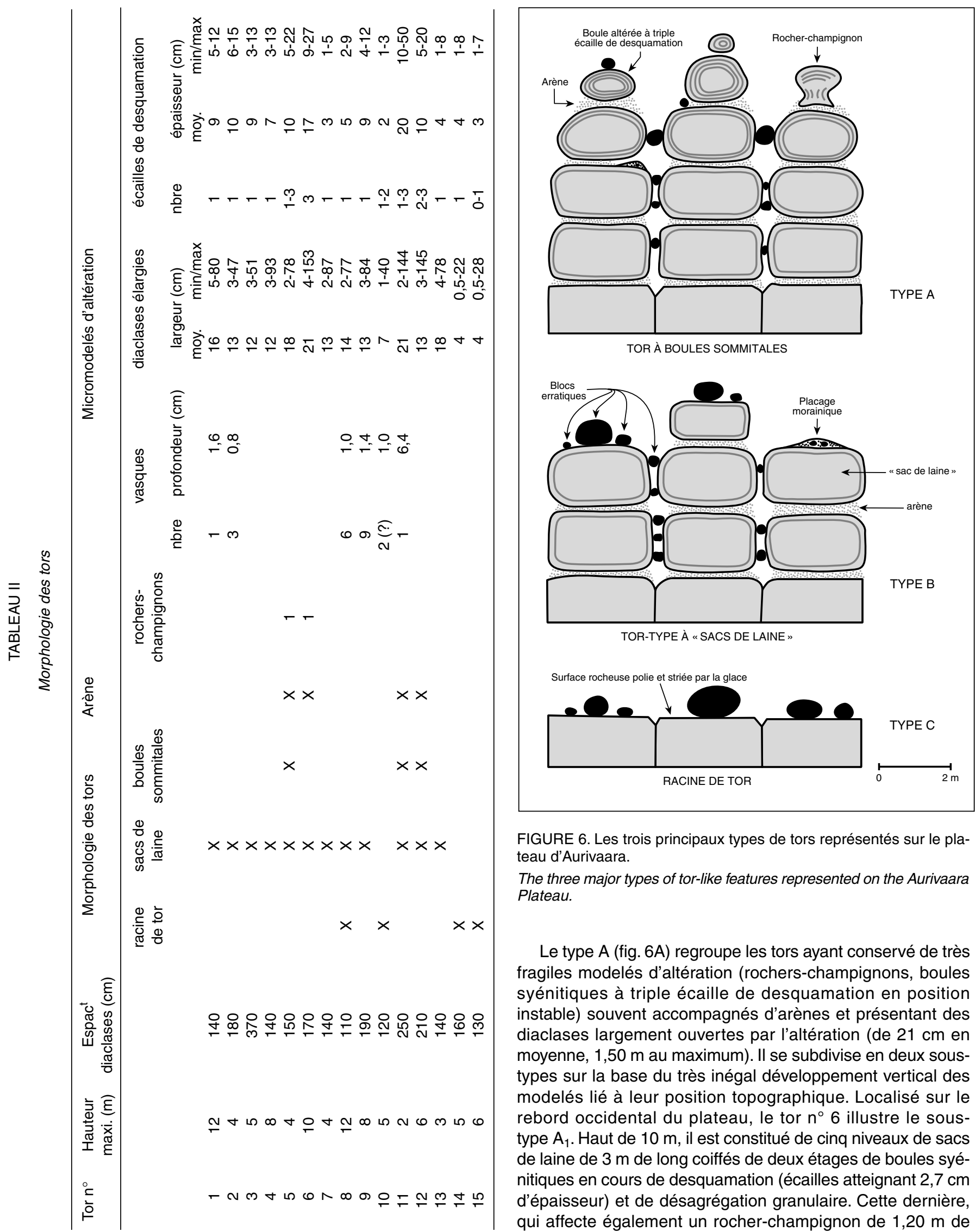

FIGURE 6. Les trois principaux types de tors représentés sur le plateau d'Aurivaara.

The three major types of tor-like features represented on the Aurivaara Plateau.

Le type A (fig. $6 \mathrm{~A}$ ) regroupe les tors ayant conservé de très fragiles modelés d'altération (rochers-champignons, boules syénitiques à triple écaille de desquamation en position instable) souvent accompagnés d'arènes et présentant des diaclases largement ouvertes par l'altération (de $21 \mathrm{~cm}$ en moyenne, 1,50 $\mathrm{m}$ au maximum). II se subdivise en deux soustypes sur la base du très inégal développement vertical des modelés lié à leur position topographique. Localisé sur le rebord occidental du plateau, le tor $n^{\circ} 6$ illustre le soustype $A_{1}$. Haut de $10 \mathrm{~m}$, il est constitué de cinq niveaux de sacs de laine de $3 \mathrm{~m}$ de long coiffés de deux étages de boules syénitiques en cours de desquamation (écailles atteignant $2,7 \mathrm{~cm}$ d'épaisseur) et de désagrégation granulaire. Cette dernière, qui affecte également un rocher-champignon de 1,20 $\mathrm{m}$ de 
haut, se traduit par la présence localisée d'arènes très grossières (à l'image des microclines qui en constituent la masse), qui apparaissent saupoudrées de sables allochtones; dans un site analogue visité en 1992, une vasque de $70 \mathrm{~cm}$ de long et de $11 \mathrm{~cm}$ de profondeur avait été observée. Illustré par les tors $n^{\circ} 5,11$ et 12 , le sous-type $A_{2}$ présente les mêmes micromodelés et formations d'altération que le type $A_{1}$ (incluant une pierre branlante dont les trois écailles de desquamation atteignent chacune jusqu'à $5 \mathrm{~cm}$ d'épaisseur), mais s'en différencie par son faible développement vertical (2-4 m). Celui-ci s'explique par une situation très interne, au cœur du plateau d'Aurivaara, moins favorable que la rupture de pente bordière à l'exhumation des cœurs rocheux. Les tors apparaissent ici encore enterrés dans leurs arènes qu'il n'a toutefois pas été possible d'observer sur plus de $20 \mathrm{~cm}$ d'épaisseur (dans une diaclase).

Le type $B$ (fig. 6B) est, de loin, le mieux représenté dans le site d'étude où il atteint 4 à $12 \mathrm{~m}$ de hauteur, et est généralement constitué de deux à quatre étages de sacs de laine de $3 \mathrm{~m}$ de long aux angles arrondis. Une écaille de desquamation de $5 \mathrm{~mm}$ à $1 \mathrm{~cm}$ d'épaisseur est fréquemment visible à leur surface. Quant aux diaclases séparant les sacs de laine, elles sont ouvertes de $16 \mathrm{~cm}$ en moyenne.

Le type $C$ (fig. $6 \mathrm{C}$ ) est illustré par cinq tors, tous situés à proximité du rebord oriental du plateau d'Aurivaara, qui sont réduits à l'état de racines et portent les témoignages d'un récurage, voire d'un profilage par la glace. Le type $\mathrm{C}_{1}$ (tors $\mathrm{n}^{\circ} 8$ à 10) associe des sacs de laine analogues à ceux caractérisant le type $A$ et de véritables entablements polis et striés (fig. 7) dont l'état de surface rappelle celui observé sur les roches moutonnées syénitiques libérées des glaces vers 10000 ans BP à proximité de la frontière norvégienne (André, 1995). Diaclases pratiquement fermées, rares vasques à peine creusées $(<2 \mathrm{~cm})$, phénocristaux polis légèrement en saillie et desquamation millimétrique portent témoignage du caractère pelliculaire d'une météorisation manifestement holocène. Dans le même secteur, des sacs de laine issus des tors ont été apparemment déplacés à contrepente, donc par la glace, sur quelques dizaines de mètres (sur des pentes infimes, de l'ordre de $1-2^{\circ}$ ), toujours en direction de l'ouest (NNO à SSO). Plus au nord, les racines de tor apparaissent profilées en roches moutonnées mal venues car non guidées par la structure, et dominent un paysage bosselé en verrous et ombilics. Nez rocheux et stries indiquent un écoulement de la glace en direction de l'ouest (mais avec d'importantes variations du NO au SO).

\section{ÉTAT D'ALTÉRATION DU MATÉRIEL AUTOCHTONE ET ALLOCHTONE}

L'étude macroscopique et microscopique des échantillons rocheux apporte des éléments d'information intéressants sur l'état d'altération des matériaux constitutifs des tors et des dépôts glaciaires voilant le plateau d'Aurivaara.

\section{ÉTAT D'ALTÉRATION DES SYÉNITES IN SITU}

Les premières observations effectuées sur les arènes associées aux tors n'apportent guère d'éléments susceptibles d'étayer l'hypothèse de l'ancienneté de l'altération responsable de la préformation des tors. Si des volumes d'arènes épaisses ou évoluées sont peut-être dissimulés sous le revêtement morainique qui s'épaissit entre les tors, ces derniers ne laissent voir que de très discontinues formations d'altération in situ, dont l'épaisseur maximale $(20 \mathrm{~cm})$ excède certes, la très pelliculaire arénisation holocène des syénites (Dahl, 1967; André, 1995),

FIGURE 7. Racine de tor polie par la glace entre les tors $n^{\circ s} 8$ et 9 (rebord oriental du plateau d'Aurivaara). Cliché M.-F. A.

Ice-polished tor root between tors Nos. 8 and 9 (eastern edge of the Aurivaara Plateau).

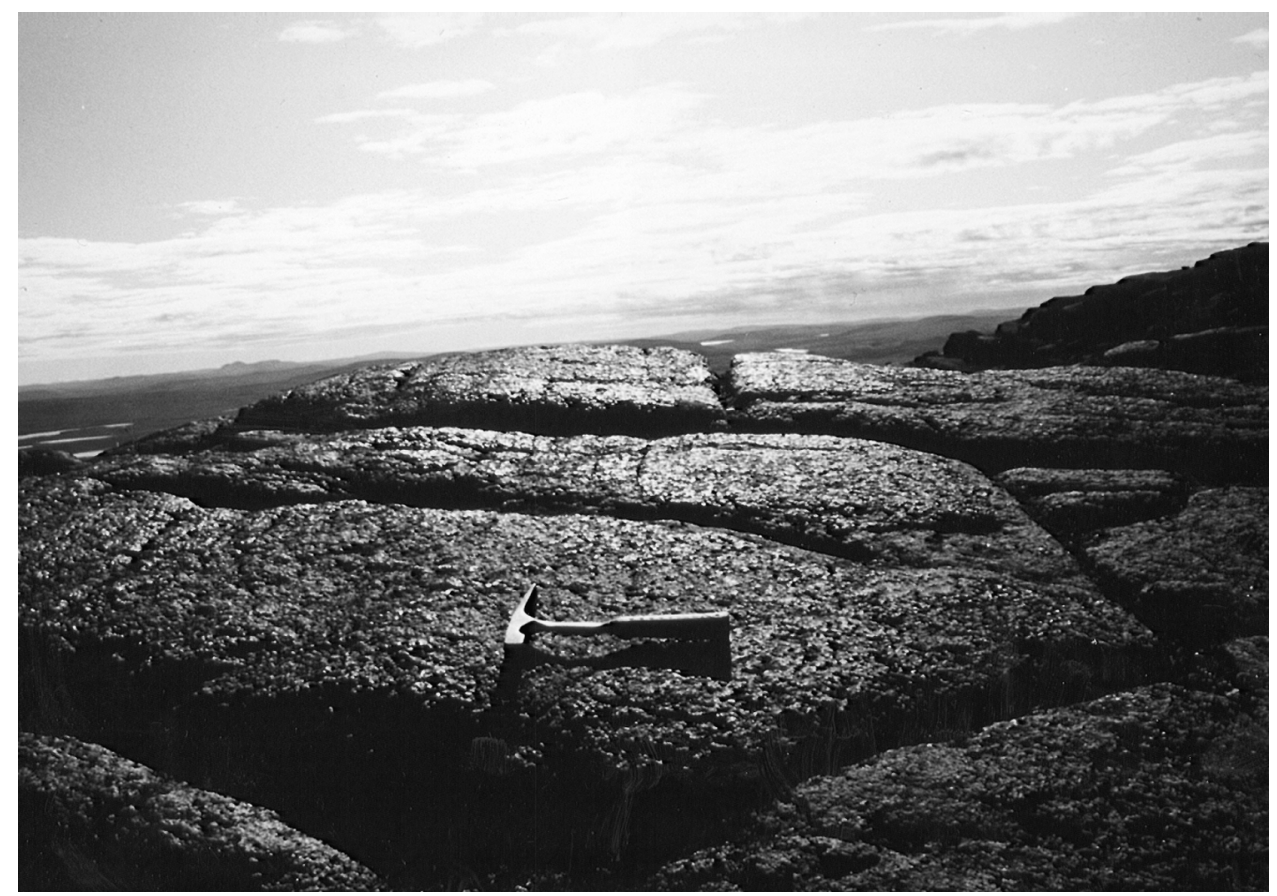


mais dont la granulométrie très sableuse témoigne, a priori, d'une arénisation ménagée même si, en surface, son caractère grossier a naturellement été accentué par le vannage éolien, très actif au sommet du plateau. Le seul échantillon atypique (le $\mathrm{n}^{\circ} 55$ ), de texture plus fine, limono-argileuse, a été prélevé au fond d'une diaclase et J. Dejou (communication personnelle, 2000) y voit la simple expression d'une différenciation texturale bien connue entre arène classique et arène ayant bénéficié de l'activation du drainage au niveau des diaclases. La rareté et le faible degré d'évolution des arènes associées aux tors ne sont guère surprenants dans la mesure où la massiveté des tors en fait les noyaux durs de l'altération différentielle. Si arènes évoluées il y a, elles sont à rechercher sous les placages morainiques déposés entre les tors. Encore n'est-il pas sûr d'y trouver des formations plus évoluées qu'en surface dans la mesure où les altérites épaisses retrouvées ailleurs dans cette position ne représentent bien souvent que les racines de profils d'altération dont le sommet plus évolué a été évacué par les glaciers (Godard, 1989).

Si l'analyse des arènes s'avère peu concluante, il en va différemment des observations effectuées sur l'état d'altération des boules rocheuses constitutives des tors, particulièrement fécondes si on les compare aux effets de la seule météorisation holocène sur les roches moutonnées syénitiques voisines de Laponie norvégienne. Certes, l'interprétation des lames minces requiert de faire la part de l'altération deutérique et de l'altération météorique, cette dernière étant la seule à prendre en considération pour notre propos. Parmi les manifestations de l'altération profonde, qui s'inscrivent dans le cadre d'évolutions magmatiques et métamorphiques, ont été identifiées par B. Azambre, de l'Université Paris VI, et C. Le
Cœur, de l'Université Paris I - Laboratoire «Pierre Birot » de Meudon (communication personnelle, 2000) : la chloritisation et la kaolinisation des feldspaths (qui leur confèrent un aspect trouble) et le remplacement de certains pyroxènes par des minéraux opaques (dont certains sont automorphes) ou par des amphiboles secondaires et des produits micacés. En revanche, la très large diffusion des oxydes de fer, qui pénètrent le réseau microfissural et s'insinuent dans les plans de clivage des pyroxènes et les joints intergranulaires (effets de bordure), peut être considérée comme l'expression d'une altération météorique des pyroxènes et des opaques. Il en va sans doute de même de l'ouralitisation des pyroxènes qui évoluent vers la hornblende par hydratation.

Si elle n'est l'indice que d'une altération ménagée, cette pénétration du réseau cristallin par les oxydes de fer ne se retrouve nullement dans les syénites précambriennes de la fenêtre de Rombak, dans les Calédonides, qui arment les roches moutonnées weichseliennes. Sur les lames minces prélevées au col de Riksgränsen-Björnfjell, on ne trouve aucune trace d'oxydes de fer dans le réseau microfissural, pourtant bien développé. Cette différence notable entre l'état des syénites constitutives des tors de Laponie suédoise et celui des syénites des roches moutonnées de Laponie norvégienne laisse à penser qu'à Aurivaara, nous nous trouvons en présence de volumes résiduels d'une altération ancienne, ce que confirme l'étude comparée de la largeur des diaclases (tabl. III).

Sur les roches moutonnées de Björnfjell, en effet, les mesures effectuées font apparaître un élargissement moyen des diaclases de $2 \mathrm{~cm}$ en 10000 ans (André, 1995). Cette lenteur est à mettre sur le compte de la pente des «dos de baleine » profilés par la glace, qui favorise un ressuyage rapide des

TABLEAU III

Élargissement des diaclases et durée d'évolution : contraste marqué entre les tors préglaciaires d'Aurivaara et les roches moutonnées tardi-weichseliennes de Riksgränsen (Laponie suédoise et norvégienne)

\begin{tabular}{|c|c|c|c|c|}
\hline Localisation & Type de modelé & \multicolumn{2}{|c|}{ Largeur des diaclases $(\mathrm{mm})$} & Source \\
\hline \multirow{7}{*}{$\begin{array}{l}\text { Riksgränsen-Björnfjell, } 68^{\circ} \mathrm{N} \\
\text { (Laponie norvégienne) }\end{array}$} & & moy. & mini-maxi & \\
\hline & roche moutonnée & 2,5 & $0,8-6$ & \\
\hline & roche moutonnée & 3,0 & 0,5-12 & André,1995 \\
\hline & roche moutonnée & 2,7 & $0,2-14$ & \\
\hline & roche moutonnée & 2,3 & $0,5-11$ & \\
\hline & roche moutonnée & 2,8 & $0,1-10$ & \\
\hline & roche moutonnée & 2,7 & $0,4-11$ & \\
\hline \multirow{11}{*}{$\begin{array}{l}\text { Aurivaara, } 68^{\circ} \mathrm{N} \\
\text { (Laponie suédoise) }\end{array}$} & racine de tor profilée & & & \\
\hline & en roche moutonnée & 4 & $0,5-22$ & André, cette étude \\
\hline & id. & 4 & $0,5-28$ & \\
\hline & racine de tor polie & 7 & $1-40$ & \\
\hline & tor à «sacs de laine» & 16 & $5-80$ & \\
\hline & id. & 13 & $3-47$ & \\
\hline & $i d$. & 12 & $3-51$ & \\
\hline & id. & 12 & $3-93$ & \\
\hline & tor à boules altérées & 13 & 3-145 & \\
\hline & id. & 21 & $2-144$ & \\
\hline & $i d$. & 21 & $4-153$ & \\
\hline
\end{tabular}


affleurements, empêche la neige d'y séjourner et n'autorise, le long des diaclases comme en surface, qu'une très pelliculaire désagrégation granulaire biogénique. Les sites étudiés se situent en effet à l'écart des sites marécageux et tourbeux où une meilleure alimentation en eau est à l'origine d'un soulèvement gélival qui écarte mécaniquement les lèvres des diaclases.

Sur les tors d'Aurivaara, l'hétérogénéité est beaucoup plus grande et la largeur moyenne des diaclases ouvertes par la météorisation $(13 \mathrm{~cm})$ intègre des valeurs très disparates qui témoignent à coup sûr de la diversité des durées d'évolution. Si l'on cherche à estimer l'ancienneté des tors, il apparaît préférable de ne prendre en compte que les valeurs maximales relevées à la surface des tors ayant conservé leur cortège de micromodelés d'altération : la largeur maximale des diaclases y est de 1,50 m et, si l'on retient la vitesse holocène d'ouverture des diaclases de $2 \mathrm{~mm} \mathrm{ka}^{-1}$ comme un ordre de grandeur acceptable, ce sont près de 800000 ans qui ont été nécessaires à l'ouverture de telles diaclases.

\section{ÉTAT D'ALTÉRATION DES DÉPÔTS GLACIAIRES}

À quelques mètres de distance, voire côte à côte, les erratiques du plateau d'Aurivaara présentent des états d'altération d'une grande diversité et ce, quelle que soit leur composition pétrographique. Certains blocs de granite rose à grain moyen apparaissent d'une très grande fraîcheur, cependant que d'autres montrent une légère mordorisation des biotites, d'autres enfin prenant la forme de boules présentant deux écailles de desquamation de plus d'un centimètre d'épaisseur. II en va de même des granites blancs à grain fin à moyen qui peuvent apparaître parfaitement sains, en cours de desquamation (écaille de $2 \mathrm{~mm}$ d'épaisseur), ou «pourris» dans la masse et débités sur place en «tranches de saucisson». La diversité est également de règle pour les roches très quartzeuses finement cristallisées (mylonites et quartzites) dont certaines sont d'une extrême fraîcheur, cependant que d'autres présentent un cortex rouge épais de $8 \mathrm{~mm}$ et que d'autres, enfin, profondément cariées, se brisent aisément au marteau.

Ce très inégal degré d'altération contraste avec la fraîcheur d'ensemble des erratiques qui coiffent les roches moutonnées weichseliennes observées sur le col qui sépare le bassin du lac Torneträsk du Rombakfjord conduisant à Narvik. Contrairement à ce col puissamment récuré lors du dernier épisode glaciaire, le plateau d'Aurivaara a manifestement conservé les traces de plusieurs phases d'englacement, ce que confirme l'examen des matrices morainiques prélevées sur les tors et dans les diaclases. Comme le montrent les courbes granulométriques (fig. 8), le contraste est grand entre la fraîcheur de la matrice sableuse des placages superficiels (éch. 37,38 et 43, prélevés à $5 \mathrm{~cm}$ de profondeur) et l'altération dont témoigne la matrice limono-argileuse (56\% de silts et d'argiles) du matériel morainique remonté en surface à la faveur d'ostioles entre les tors 5 et 9 (le $\left.n^{\circ} 36\right)$. Mais un tel contraste est rarement observé, soit que la dernière génération — d'une grande fraîcheur — voile les apports antérieurs, soit qu'un mélange d'apports se soit opéré, la formation morainique prélevée étant alors polygénique. Un tel mélange est d'ailleurs attesté dans les remplissages des diaclases de certains tors (tor $n^{\circ} 9$ notamment) où de gros erratiques anciens (très altérés) sont perchés au-dessus de petits blocs allochtones d'une grande fraîcheur qui ont glissé au fond des diaclases. La difficulté de séparer avec certitude les diverses générations de dépôts glaciaires se complique du fait des remaniements successifs. En témoignent quelques erratiques granitiques polis en surface mais «pourris à cœur », empruntés sans doute à d'anciens placages morainiques (ou à des racines d'altérites), et transportés à l'état gelé par l'inlandsis scandinave.

\section{INTERPRÉTATION ET DISCUSSION}

L'étude du site d'Aurivaara apporte des éléments de réponse à trois questions fondamentales : l'âge des tors présents dans les socles englacés, les modalités de la dynamique glaciaire et l'épaisseur de la tranche d'ablation glaciaire quaternaire.

\section{ÂGE DES TORS ET IMPLICATIONS GLACIOLOGIQUES GÉNÉRALES}

En Laponie suédoise, la présence de dépôts glaciaires polygéniques dans les diaclases des tors et à leur surface constitue

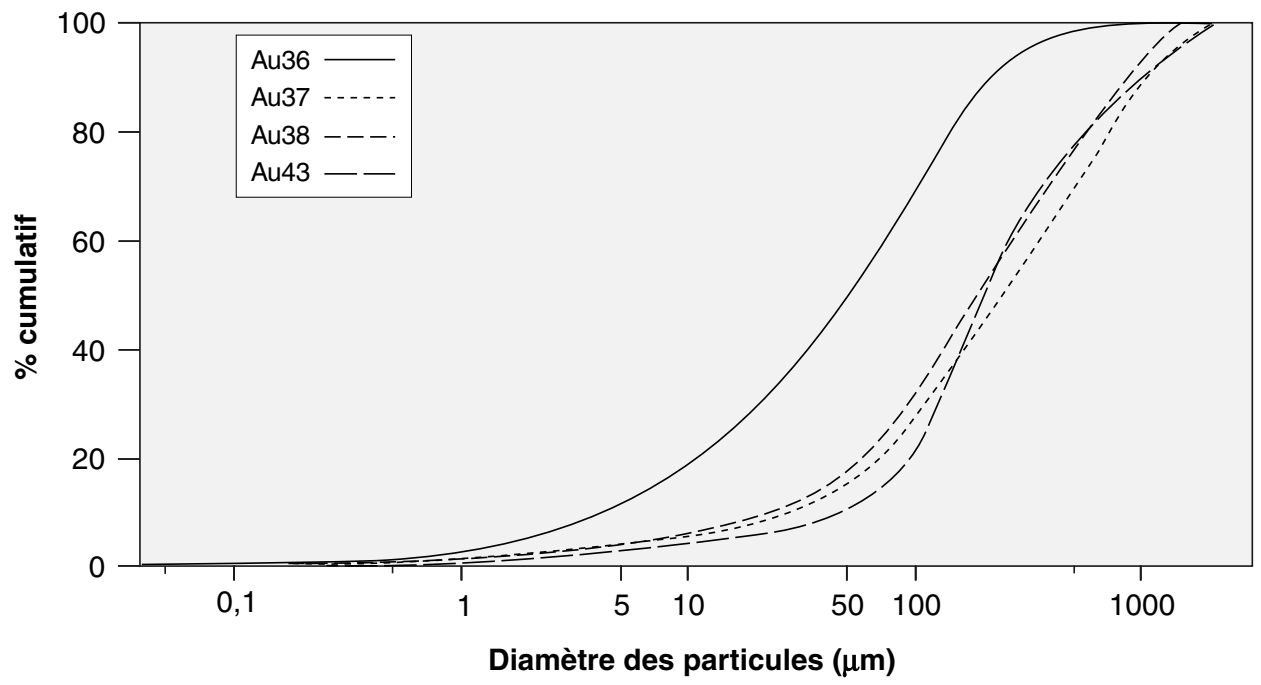

FIGURE 8. Courbes granulométriques des matrices morainiques. La médiane de la moraine profonde $\mathrm{Au}$ $36($ ca $50 \mu \mathrm{m})$ contraste fortement avec celle des autres échantillons provenant de placages superficiels très frais $(c a 200 \mu \mathrm{m})$.

Grain-size distribution of morainic matrixes. The median of the deep till deposit Au 36 (ca $50 \mu \mathrm{m}$ ) strongly contrasts with the one of the other samples belonging to very fresh and superficial till deposits (ca $200 \mu \mathrm{m}$ ). 
un indice de l'ancienneté de ces modelés de déchaussement. Cette hypothèse est confirmée par la largeur de leurs diaclases (max. 1,50 m) qui laisse présager une durée d'altération avoisinant le million d'années. Celle-ci, qui excède la durée des périodes interglaciaires cumulées, renvoie donc à l'ère tertiaire la «préparation » des tors par cryptodécomposition. Mais qu'en est-il de l'âge du déchaussement de ces modelés et donc de leur apparition dans le paysage? Un âge tardi-tertiaire est envisageable dans la mesure où nombre de ces tors, comme ceux du Dartmoor, entrent en contact à la faveur d'un véritable knick avec des topographies planes au profil extrêmement tendu, qui évoquent des éléments de pédiplaine à inselbergs hérités des aplanissements tertiaires. Parfois envisagée, la responsabilité de la cryoplanation dans l'élaboration de tels plans doit aujourd'hui être écartée tant les doutes se multiplient quant à l'efficacité géomorphologique de ce processus. On pense notamment aux travaux de French et Harry (1992) sur les pédiments du Yukon et de Migon (1997) sur les strandflats du Spitsberg.

Compte tenu de sa position septentrionale, le plateau d'Aurivaara a très vraisemblablement passé l'essentiel du Quaternaire sous la glace, d'autant qu'il était situé non loin du dôme principal de l'inlandsis scandinave. Au sommet des tors, l'association - dans le même plan — de fragiles micromodelés d'altération in situ et de gros blocs erratiques d'une extrême fraîcheur, ne saurait s'expliquer que par le recouvrement du plateau par un inlandsis à base froide. En Suède, le rôle protecteur d'un tel glacier a été précédemment mis en évidence par les chercheurs scandinaves (Lagerbäck, 1988; Kleman et Borgström, 1990 et 1994 ; Hättestrand, 1994 et 1998; Clarhäll et Kleman, 1999).

Élaboré par Rapp (1996) à partir d'observations effectuées par les chercheurs sur divers plateaux scandinaves, le schéma explicatif présenté sur la figure 9 nous paraît le seul modèle susceptible de rendre compte du dispositif incongru observé à Aurivaara : à la base de l'Inlandsis scandinave, une semelle froide aurait emprisonné dans une gangue de glace les modelés de déchaussement, cependant qu'au-dessus voyageaient les futurs blocs erratiques de provenance orientale, qui auraient été «parachutés » sur et entre les tors lors de la déglaciation. L'existence d'un régime glaciaire à base froide est d'ailleurs également attesté dans le site d'étude par la présence d'erratiques inégalement altérés dans les minces champs de blocaille que les glaciers quaternaires ont été manifestement impuissants à déblayer. En matière de dynamique, la dominante qui se dégage de l'étude du site d'Aurivaara est celle d'un processus cumulatif de dépôt glaciaire et fluvioglaciaire, opérant en fin d'épisode glaciaire, et allant de pair avec le respect des modelés préexistants, durablement scellés par la chape basale de glace froide de l'Inlandsis scandinave. La prédominance manifeste de l'accumulation sur l'ablation dans le site étudié est en prise avec la thèse du « palimpseste glaciaire », avancée par l'équipe de Kleman (1992) qui a montré en divers sites que le dernier épisode glaciaire n'avait nullement effacé les traces des épisodes antérieurs, de nombreux plateaux scandinaves portant des dépôts glaciaires et fluvioglaciaires polygéniques.

Le site d'Aurivaara offre également une nouvelle illustration de la théorie de l'érosion glaciaire sélective élaborée par Sugden (1978), dans la mesure où ce plateau, pratiquement intouché, voisine avec l'ombilic de surcreusement du lac Torneträsk qui succède au verrou à roches moutonnées marquant la frontière entre la Suède et la Norvège. Le plateau d'Aurivaara possède d'ailleurs tous les attributs des «îlots de glace à base froide » identifiés sur les hautes terres scandinaves par Kleman et Borgström (1994) puisqu'ont été
FIGURE 9. Schéma explicatif de la coexistence des tors et des erratiques (d'après Rapp, 1996). Au sommet, glace en mouvement transportant les futurs blocs erratiques; à la base, chape de glace froide scellant la topographie à tors et champs de blocaille.

Sketch explaining the co-existence of tor-like features and erratics (after Rapp, 1996). On top, mobile ice carrying the future erratic blocks; at the base, cold ice protecting the subglacial topography with tors and felsenmeers.

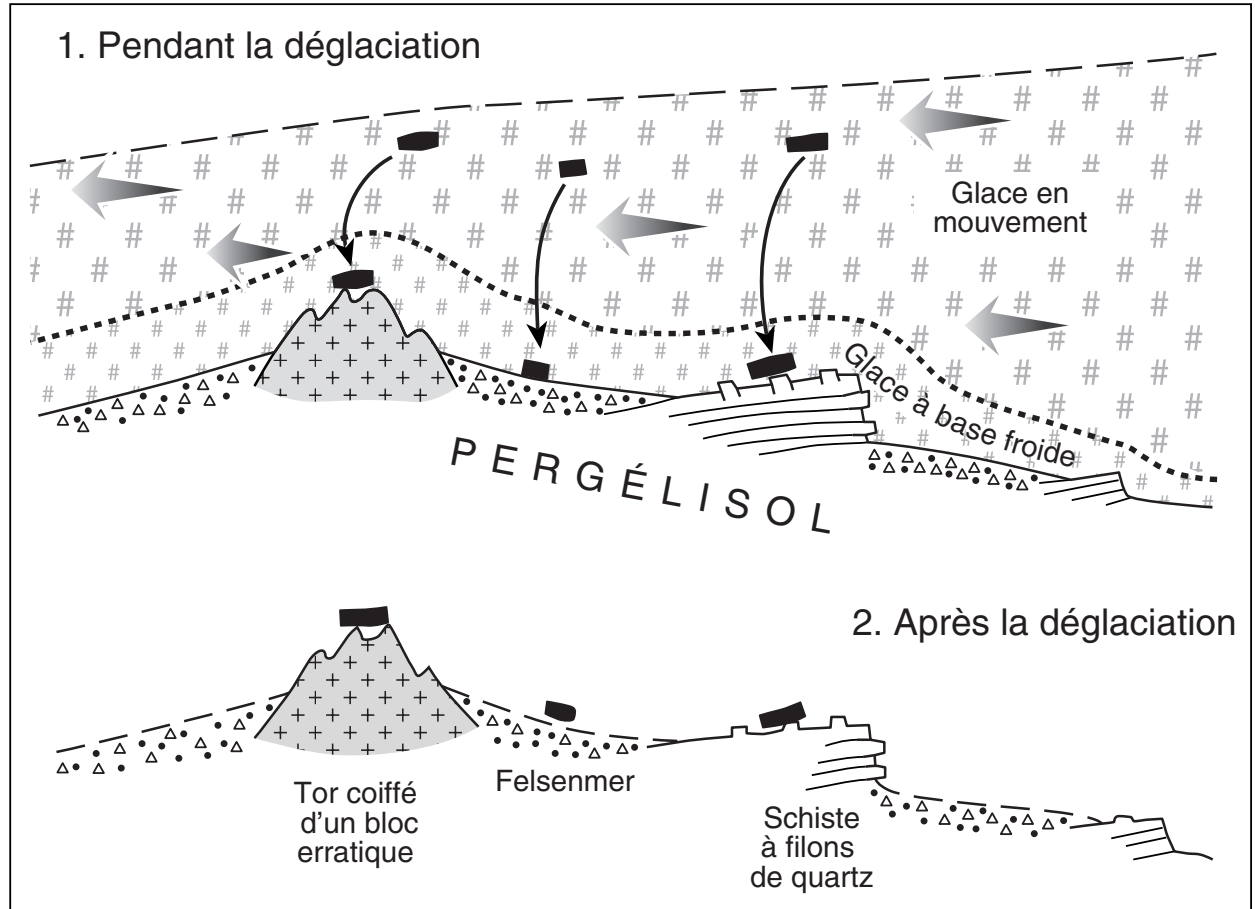


préservés à sa surface des modelés de type tor, des champs de blocaille (certes circonscrits) et des sols figurés élaborés aux dépens de placages morainiques anciens. Peut-être la chape protectrice s'est-elle formée dans le cadre d'un englacement « instantané » des hautes terres scandinaves, à l'image de la rapide formation de vastes névés sur les plateaux du Québec-Labrador au Petit Âge glaciaire (Andrews et al., 1976). Bien connues aujourd'hui dans le Haut-Arctique, de telles chapes de glace froide, qu'elles s'inscrivent dans un englacement local, de type fonna ou «calotte », ou qu'elles constituent in fine la semelle soudée au pergélisol d'un inlandsis, ont la propriété de conserver des formes et des formations - minérales ou végétales - extrêmement fragiles que le retrait glaciaire contemporain met progressivement au jour : cordons littoraux au Svalbard (Jonsson, 1983), réseaux polygonaux en terres de Baffin et d'Ellesmere (Falconer, 1966; England, 1986), et même végétation de toundra - avec thalles lichéniques non déformés - au Groenland, dans l'archipel nord-canadien et au Svalbard (Goldthwait, 1960 ; Falconer, 1966; Bergsma et al., 1984 ; Holmgren, 1984). Dans un tel contexte, la conservation de tors et de felsenmeers ne soulève donc pas le moindre problème.

\section{DU TOR À LA ROCHE MOUTONNÉE : UN CONTINUUM DYNAMIQUE TRADUISANT UNE EFFICACITÉ CROISSANTE DE L'ÉROSION GLACIAIRE}

On ne saurait s'en tenir, s'agissant du secteur d'étude, à la vision monolithique d'un inlandsis scandinave à base froide, dans la mesure où les quinze modelés de déchaussement présents sur le plateau d'Aurivaara n'ont pas été également respectés par la glace. Le contraste est, en effet, frappant entre les empilements de boules altérées et de rochers-champignons préservés au sud-ouest et les racines de tor polies, voire profilées par la glace, au nord-est (fig. 7, voir plus haut). Doit-on voir dans ce dispositif l'expression d'un régime thermique fon- damentalement contrasté (à base chaude au NE, à base froide au SO) ? Si oui, la différence de régime thermique est-elle à mettre en relation avec la position des affleurements par rapport au sens d'écoulement des glaces (les tors du NE étant en première ligne par rapport au flux glaciaire lors du maximum glaciaire, les tors du SO se situant en position d'abri)? Compte tenu de la grande variabilité spatio-temporelle du régime thermique des inlandsis au long du Quaternaire, on est en droit de considérer que le contraste observé sur le plateau d'Aurivaara n'exprime qu'une tendance, n'est que la résultante d'une succession de phases d'englacement qui se sont révélées, in fine, majoritairement protectrices au SO (glace froide), plus érosives au NE où les tors, réduits à l'état de racines polies et striées, portent témoignage d'un régime basal au moins temporairement à base chaude.

Mais si le contraste NE/SO est une réalité, on n'aurait garde d'oublier que tous les stades de transition existent entre le tor bien conservé et sa racine profilée en roche moutonnée (fig. 10) et que c'est finalement un type intermédiaire, celui du tor décoiffé à sacs de laine qui est le mieux représenté sur les plateaux lapons. Nul équivalent, à cette échelle, du zonage très clair établi par Dyke (1993) dans l'archipel nord-canadien où les paysages se distribuent aisément entre zones décapées et non décapées par la glace. Sans doute parce que le plateau d'Aurivaara ne s'intègre pas dans un modèle d'englacement simple, de type «calotte », applicable dans les îles de l'archipel nord-canadien, mais doit être replacé dans celui d'un inlandsis complexe à la fois dans son régime thermique et dans sa géométrie (Kleman 1992; Kleman et Borgström, 1994 ; Kleman et al., 1999).

\section{L'APPORT DES TORS À L'ÉVALUATION DE LA TRANCHE D'ABLATION GLACIAIRE QUATERNAIRE}

Si le gradient d'efficacité croissante de l'érosion glaciaire qui se traduit par le continuum dynamique pouvant conduire

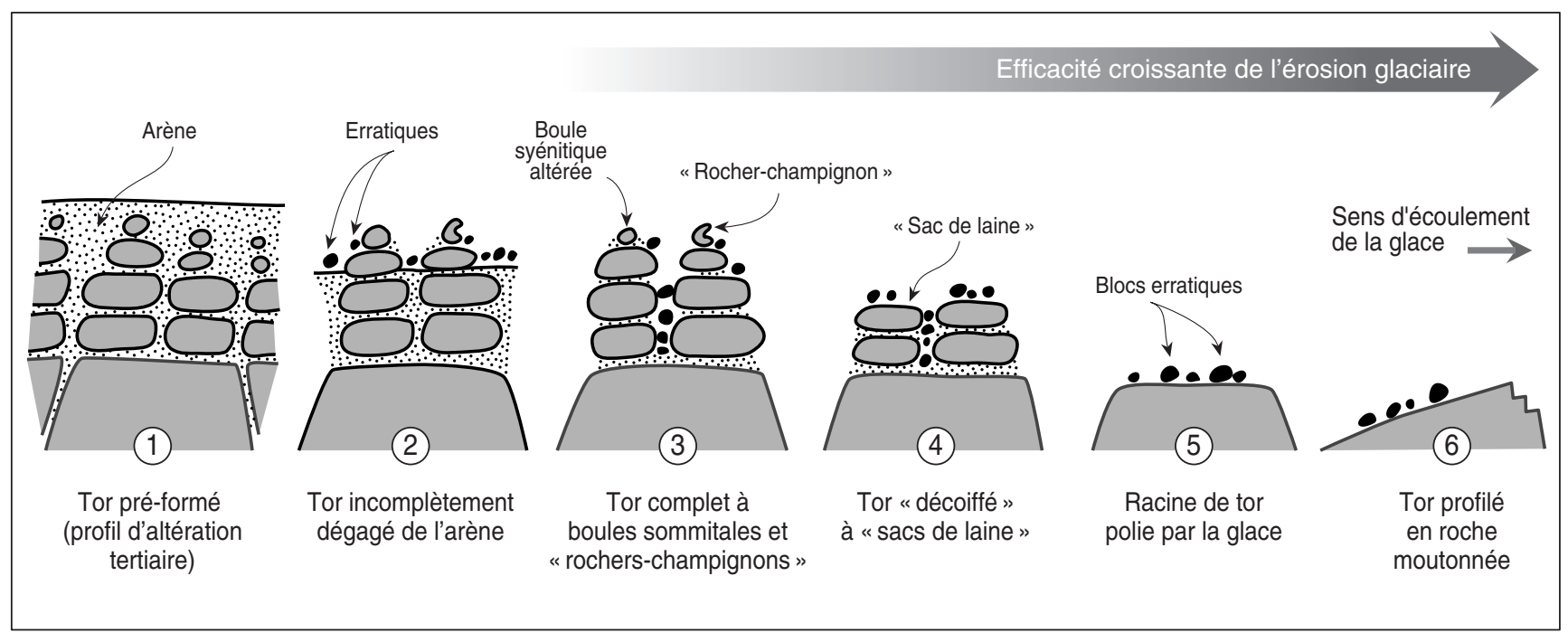

FIGURE 10. Du tor à la roche moutonnée : un continuum dynamique. Les types 3 à 6 se rencontrent en situation périphérique (rebord de plateau), le type 2 en situation interne (cœur de plateau).

From the well preserved tor to the roche moutonnée: a dynamic continuum. Types 3 to 6 can be seen on the plateau edges, and type 2 in the inner part. 
du tor à la roche moutonnée (fig. 10) n'est que bien imparfaitement expliqué par la cinématique glaciaire, il peut être légitimement utilisé dans la perspective d'une quantification de l'érosion glaciaire quaternaire.

Doit-on parler d'une érosion glaciaire nulle dès lors que l'échafaudage des tors apparaît complet, c'est-à-dire qu'il comporte des micromodelés d'altération sommitaux (pierres branlantes à triple écaille de desquamation et «rochers champignon », voir plus haut fig. 6A)? Rien n'est moins sûr dans la mesure où le sommet de ces tors ne porte pas, contrairement aux tors anciennement englacés des Highlands d'Écosse et des Mourne Mountains d'Irlande du Nord (Sugden, 1968; Le Cœur, 1980), des vasques de profondeur métrique remontant au Tertiaire ${ }^{2}$. Certes, cette absence peut être imputée à l'exiguïté des surfaces sommitales, souvent dépourvues d'éléments plans propices à la formation - et à la conservation - des vasques; une telle explication ne saurait être complètement écartée dans la mesure où les vastes « racines » de tor rafraîchies par l'inlandsis weichselien portent déjà quelques vasques de profondeur centimétrique. Pour autant, l'absence généralisée de vasques au sommet des tors les plus achevés laisse à penser qu'ils ont été décapités par l'érosion glaciaire. Un « décoiffage » qui peut avoir été effectué, y compris dans le cadre d'un englacement à base froide, dès lors que la semelle froide était mince et que la glace en mouvement surincombante était chargée à sa base de gros blocs susceptibles d'entrer en collision avec le sommet des modelés de déchaussement.

Un autre indice du décoiffage des tors par l'érosion glaciaire réside dans le développement vertical généralement modeste des tors d'Aurivaara. Si l'on élimine les tors du cœur du plateau encore empâtés dans leurs arènes, les modelés de rebord de plateau n'excèdent jamais $12 \mathrm{~m}$ de hauteur, l'ordre de grandeur le plus courant se situant autour de $6 \mathrm{~m}$. On est loin des $22 \mathrm{~m}$ de hauteur des Ben Avon Tors, dans les Cairngorms (Highlands d'Écosse), qui portent à leur sommet des vasques de $2 \mathrm{~m}$ de profondeur témoignant du degré zéro de l'érosion glaciaire. Compte tenu de la moindre énergie du relief dans la région étudiée et de la hauteur des tors bien conservés en domaine non englacé (Dartmoor), il est vraisemblable que la hauteur maximale des tors d'Aurivaara n'a jamais excédé une quinzaine de mètres. Sur la base de la hauteur actuelle des modelés, la tranche d'ablation glaciaire se situerait donc dans une fourchette de 3 à $12 \mathrm{~m}$, la valeur la plus élevée correspondant aux secteurs d'impact glaciaire maximal, avec réduction des tors à l'état de racines.

Encore faut-il prendre en compte les éléments de topographie pédimentaire conservés entre les tors, à peine voilées d'un très mince revêtement morainique ou de nappes de blocs issues des affleurements de syénite rose densément diaclasée. La perfection de ces surfaces héritées du Tertiaire, qui s'adossent aux tors à la faveur d'un véritable knick, laisse

2. Quand on sait que la vitesse de creusement des vasques fut à l'Holocène de 2 à $3,5 \mathrm{~mm} \mathrm{ka}^{-1}$ dans le socle scandinave (André, 1995), c'est une durée de l'ordre du million d'années qu'il faut envisager pour donner naissance aux vasques de $2 \mathrm{~m}$ de profondeur décrites au sommet des Ben Avon tors, dans les Cairngorms, par Ballantyne (1994). à penser que là où elles sont conservées, la tranche d'ablation glaciaire peut être considérée comme nulle. En revanche, là où apparaissent, en contrebas de ces éléments de surface, de légers vallonnements (dénivelés de l'ordre de $5 \mathrm{~m}$ ), ceux-ci témoignent d'une ablation glaciaire d'ordre métrique, au mieux plurimétrique, mais peut-être proche de zéro. En effet, il n'est pas déraisonnable d'émettre l'hypothèse que ces vallonnements étaient déjà en place - du moins en partie - à la fin du Tertiaire et qu'ils ont été le siège d'une accumulation plus que d'une ablation glaciaire.

D'une manière générale, la discrétion des retouches glaciaires sur le plateau d'Aurivaara contraste avec le surcreusement notable opéré le long de l'entaille majeure la plus proche, celle qui recèle l'ombilic du lac Torneträsk, d'une profondeur maximale de 189 m d'après les derniers échosondages (communication écrite du 14 avril 2000 de Nils Åke Andersson, Abisko Scientific Research Station, Laponie suédoise). Mais la tranche d'ablation glaciaire proposée dans la région d'étude (0-12 $\mathrm{m}$ ) est également à confronter aux estimations publiées dans d'autres régions de socles englacés. Par delà l'estimation très globale de Ruddiman (1977) pour l'ensemble de la Fennoscandie (tranche moyenne érodée de $16 \mathrm{~m}$ ), les études fines conduites par Lidmar-Bergström (1997) sur la dégradation par les glaciers des surfaces infracambrienne et infracrétacée font apparaître une grande hétérogénéité spatiale; selon cet auteur, la tranche moyenne d'ablation glaciaire se situe, selon les sites, entre 0 et $30 \mathrm{~m}$. Cette hétérogénéité s'accroît si l'on prend en compte la retombée du bourrelet des Scandes sur la mer de Norvège. Dans le bassin du Sognefjord, le surcreusement atteint la valeur record de 1200 m (Nesje et Sulebak, 1994).

En Écosse, la tranche moyenne de $16 \mathrm{~m}$, déduite de la sédimentation marine au large des Hébrides et des Highlands du Nord-Ouest (Le Cœur, 1999), masque également des écarts importants entre secteurs de surcreusement (tranche d'ablation hectométrique) et plateaux intermédiaires où l'état de conservation des reliefs résiduels laisse à penser que l'abaissement de surface a été inférieur à 10 m (Le Cœur, 1994). On retrouve cet écart entre interfluves et entailles dans les Appalaches estriennes où Clément (1989), sur la base de la plus ou moins grande dégradation de la topographie préglaciaire, propose, pour une tranche d'ablation moyenne de 20-25 m, une érosion maximale de $100 \mathrm{~m}$ au niveau des entailles majeures et une érosion minimale de $6 \mathrm{~m}$ sur les plateaux intermédiaires.

\section{CONCLUSION}

L'étude du site d'Aurivaara débouche sur quatre conclusions principales :

- Un âge pré-quaternaire est proposé pour les tors, sur la base de trois critères : la très large ouverture de leurs diaclases (jusqu'à 1,50 m) impliquant une durée de météorisation voisine du million d'années; la présence dans ces diaclases de dépôts glaciaires polygéniques; l'insertion de ces modelés de déchaussement dans un système de pentes évoquant une pédiplaine à inselbergs. Une telle conclusion rejoint l'hypothèse anciennement émise par 
Linton (1955) et Sugden (1968) à propos des tors associés aux aplanissements sommitaux des Cairngorms, dans les Highlands d'Écosse, et adoptée notamment par Söderman et al. (1983), en Finlande. Elle rejoint également les observations effectuées par Dionne (1985) sur les tors schisteux de l'archipel des Nastapoka, en Hudsonie, «paléoformes ayant survécu à l'érosion glaciaire ».

- La tendance lourde à une dynamique glaciaire dominée par un régime à base froide apparaît seule à même de rendre compte de la conservation de modelés de déchaussement et fragiles microformes d'altération associées, ainsi que des minces champs de blocaille semés d'erratiques inégalement altérés qui les ceinturent. Sur le plateau d'Aurivaara, l'Inlandsis scandinave a joué un rôle fondamentalement protecteur vis-à-vis du substrat. L'extrême discrétion des manifestations de l'ablation glaciaire contraste, en effet, avec les nombreux témoignages d'un processus cumulatif de dépôt glaciaire et fluvioglaciaire opérant en fin de glaciation («parachutage» d'erratiques et de matériel morainique et fluvioglaciaire à chaque déglaciation). Cette conclusion rejoint la thèse du «palimpseste glaciaire » défendue par Kleman (1992), Hättestrand (1994), Clarhäll et Kleman (1999) et Kleman et al. (1999) sur d'autres plateaux de Suède centrale et septentrionale où ont été préservées diverses générations de dépôts glaciaires et fluvioglaciaires sous un inlandsis à base froide.

- Dans un contexte général de glace à base froide, se fait jour une extrême complexité de détail des régimes thermiques et des modalités de l'érosion glaciaire qui parvient localement à décapiter, voire à araser les empilements de boules syénitiques. L'étude des 15 tors d'Aurivaara révèle, en effet, l'existence d'un continuum dynamique conduisant du tor à la roche moutonnée, qui traduit une efficacité croissante de l'érosion glaciaire, comme l'avaient pressenti Sugden et Watts (1977), à l'île de Baffin, et Kaitanen (1989), en Laponie finlandaise. En témoigne notamment le contraste marqué entre les tors bien conservés du rebord SO du plateau d'Aurivaara et les tors arasés du NE. Ce contraste est-il à mettre sur le compte de régimes thermiques contrastés liés à la position des tors par rapport au flux glaciaire? Ou l'arasement est-il le fruit d'une érosion par de gros blocs enchâssés dans la glace en mouvement qui ont pénétré dans la semelle à base froide? Nul doute que l'existence de variations spatio-temporelles du régime thermique de l'Inlandsis scandinave, associées notamment aux modifications de sa géométrie, est à prendre en compte (Kleman et Borgström, 1994; Kleman et al. 1999).

- Enfin, les tors constituent de précieux auxiliaires dans la démarche d'évaluation de la tranche d'ablation glaciaire quaternaire. Sur le plateau d'Aurivaara, celle-ci se situerait dans une fourchette de 0 à $12 \mathrm{~m}$, la valeur la plus basse correspondant aux secteurs de conservation des éléments de pédiments à la base des tors, la plus élevée coïncidant avec les racines de tor. De tels ordres de grandeur sont en accord avec les gammes de valeur proposées dans le sud de la Scandinavie, les Appalaches estriennes et les Highlands d'Écosse par Lidmar-Bergström (1997), Clément (1989) et Le Cœur (1994, 1999). La modestie de l'ablation quaternaire en situation d'interfluve (12 m maximum sur le plateau d'Aurivaara) contraste donc avec la vigueur de l'érosion glaciaire dans les ombilics scandinaves (surcreusement voisin de $200 \mathrm{~m}$ dans le bassin du Torneträsk et supérieur à $1200 \mathrm{~m}$ dans celui du Sognefjord). L'analyse des tors du plateau d'Aurivaara vient donc conforter le modèle de l'érosion glaciaire sélective proposé par Sugden (1968, 1974, 1978) en Écosse, au Groenland et en Amérique du Nord.

\section{REMERCIEMENTS}

C'est à Anders Rapp (université de Lund) que je dois la découverte du site d'Aurivaara qu'il m'a vivement encouragé à étudier en détail. Ce fut chose faite grâce au concours financier du CNRS (UMR 6042 de Clermont-Ferrand et GDR 49 de Besançon), au soutien logistique apporté par Nils Åke Andersson (station d'Abisko, Royal Swedish Academy of Sciences) et à l'assistance efficace de Cecilia Richardson (université de Stockholm) dont le tempérament enjoué fit merveille sur le terrain dans la pluie, le vent et le brouillard. Les échantillons rocheux ont été examinés avec Bernard Azambre (Université de Paris VI), Jean Dejou (Institut national de la Recherche agronomique), Jean-Pierre Floc'h (Université de Limoges) et Charles Le Cœur (Université de Paris I et Laboratoire «Pierre Birot » de Meudon). La granulométrie laser des échantillons meubles a été effectuée au Laboratoire de Géographie physique de Clermont-Ferrand par Christèle Ballut. La réflexion menée dans le cadre de cet article a bénéficié de fructueux échanges d'informations et de publications avec Colin Ballantyne, Pierre Clément, Pierre Gangloff, Alain Godard, Clas Hättestrand, Björn Holmgren, Christer Jonasson, Veijo Kaitanen, Johan Kleman, Charles Le Cœur, Karna Lidmar-Bergström, Anders Rapp et David Sugden. C'est à bord du James Clark Ross prisonnier des glaces lors de l'été austral 1999-2000 que la rédaction de cet article a connu une avancée décisive grâce à la très stimulante musique celtique prodiguée par Robert Paterson. Les figures ont été ensuite redessinées par Jean-Pierre Magnier et la mise en forme finale du manuscrit a bénéficié de l'aide apportée par Armelle Decaulne. Le texte anglais du résumé et des légendes a été revu par Kevin Hall, et le résumé a été traduit en allemand par Agnieszka Latocha et Andreas Peterek. Mireille Bouchard, Jean-Claude Dionne, Pierre Gangloff et la Rédaction de Géographie physique et Quaternaire ont examiné avec un œil critique le manuscrit, sur la forme et sur le fond. À tous je tiens à exprimer ici ma profonde gratitude.

\section{RÉFÉRENCES}

André, M.-F., 1995. Postglacial microweathering of granite roches moutonnées in northern Scandinavia (Riksgränsen area, $68^{\circ} \mathrm{N}$ ). In O. Slaymaker, édit., Steepland Geomorphology. John Wiley \& Sons, Chichester, p. 103-127.

Andrews, J.T., Davis, P.T. et Wright, C., 1976. Little Ice Age permanent snowcover in the eastern Canadian Arctic : Extent mapped from Landsat-1 satellite imagery. Geografiska Annaler, 58A : 71-82.

Ballantyne, C.K., 1994. Scottish landform examples - 10. The tors of the Cairngorms. Scottish Geographical Magazine, $110: 54-59$.

Bennett, M.R. et Glasser, N.F., 1997. Glacial geology — ice sheets and landforms. John Wiley \& Sons, Chichester, $364 \mathrm{p}$. 
Bergsma, B.M., Svoboda, J. et Freedman, B., 1984. Entombed plant communities released by a retreating glacier at Central Ellesmere Island, Canada. Arctic, $37: 49-52$

Clarhäll, A. et Kleman, J., 1999. Distribution and glaciological implications of relict surfaces on the Ultevis plateau, northwestern Sweden. Annals of Glaciology, $28: 202-208$.

Clément, P., 1989. Processus d'érosion et évolution du relief : exemples sous climats tempérés à hivers froids (Appalaches du Québec méridional; comparaison avec la Suède centrale). Thèse d'État, Université de Lyon II, 705 p.

Dahl, R., 1967. Postglacial micro-weathering of bedrock surfaces in the Narvik district of Norway. Geografiska Annaler, 49A : 155-166.

Dionne, J.-C., 1985. Observations sur les tors des îles Bélanger et Ross, archipel des Nastapoka, Hudsonie. $53^{\circ}$ Congrès de l'Association canadiennefrançaise pour l'avancement des sciences, Chicoutimi, 20-24 mai 1985. Annales de l'ACFAS, 52-53: 174.

Dyke, A.S., 1993. Landscapes of cold-centred Late Wisconsinian ice caps, Arctic Canada. Progress in Physical Geography, 17 : 223-247.

England, J., 1986. Glacial erosion of a high arctic valley. Journal of Glaciology, $32: 60-64$.

Falconer, G., 1966. Preservation of vegetation and patterned ground under a thin ice body in northern Baffin Island, N.W.T. Geographical Bulletin, 8 : 194-200.

French, H.M. et Harry, D.G., 1992. Pediments and cold-climate conditions, Barn Mountains, unglaciated northern Yukon, Canada. Geografiska Annaler, 74A 145-157.

Gangloff, P., 1983. Les fondements géomorphologiques de la théorie des paléonunataks : le cas des Monts Torngats. Zeitschrift für Geomorphologie, Supplement Band 47 : 109-136.

Godard, A., 1966. Les tors et le problème de leur origine. Revue Géographique de l'Est, 1-2:153-170.

1989. Les vestiges des manteaux d'altération sur les socles des hautes latitudes : identification, signification. Zeitschrift für Geomorphologie, Supplement Band $72: 1-20$.

Goldthwait, R.P., 1960. Study of ice cliff in Nunatarssuaq, Greenland. Technical Report Snow Ice Permafrost Research Establishment $39: 1-103$.

Hättestrand, C., 1994. Boulder depressions in central Sweden - remnants of a pre-late Weichselian landscape? Geografiska Annaler, 76A : 153-160.

1998. The glacial geomorphology of central and northern Sweden. Sveriges Geologiska Undersökning Ca 85, $47 \mathrm{p}$

Holmgren, B., 1984. Climate and glaciation in Kong Karls Land, Eastern Svalbard, p. 291-302. In N.-A Mörner. et W. Karlén, édit., Climatic Change on a Yearly to Millenial Basis. Reidel, Dordrecht, $667 \mathrm{p}$.

Jonsson, S., 1983. On the geomorphology and past glaciation of Storöya Svalbard. Geografiska Annaler, 65A : 1-17.

Kaitanen, V., 1989. Relationships between icesheet dynamics and bedrock relief on dissected plateau areas in Finnish Lappland. Geografiska Annaler, 71A 1-15.

Kleman, J., 1990. On the use of glacial striae for reconstruction of paleo-ice sheet flow patterns. Geografiska Annaler, 72A : 217-236.

1992. The palimpsest glacial landscape in northwestern Sweden. Geografiska Annaler, 74A : 305-325.

1994. Preservation of landforms under ice sheets and ice caps. Geomorphology, $9:$ 19-32.
Kleman, J. et Borgström, I., 1990. The boulder fields of Mt. Fulufjället, westcentral Sweden — Late Weichselian boulder blankets and interstadial periglacial phenomena. Geografiska Annaler, 72A : 63-78.

Kleman, J. et Borgström, I., 1994. Glacial landforms indicative of a partly frozen bed. Journal of Glaciology, $40: 255-264$.

Kleman, J. et Hättestrand, C., 1999. Frozen-bed Fennoscandian and Laurentide ice sheets during the last glacial maximum. Nature, $402: 63-65$.

Kleman, J., Hättestrand, C. et Clarhäll, A., 1999. Zooming in on frozen-bed patches - scale-dependent controls on Fennoscandian ice sheet basal thermal zonation. Annals of Glaciology, $28: 189-194$.

Lagerbäck, R., 1988. Periglacial phenomena in the wooded areas of north Sweden — relicts from the Tärendö Interstadial. Boreas, 17 : 487-499.

Le Cœur, C., 1980. Les Mourne Mountains (Irlande du Nord) - Étude géomorphologique d'un complexe intrusif tertiaire. Thèse $3^{\mathrm{e}}$ cycle, Université de Paris I, 243 p.

1994. Évolution géomorphologique et échelles d'analyse : l'exemple des Hébrides internes (Écosse). Thèse d'État, Université de Paris I, 2 t, 766 p. 1999. Rythmes de dénudation tertiaire et quaternaire en Écosse occidentale. Géomorphologie, 4 : 291-303.

Lidmar-Bergström, K., 1997. A long-term perspective on glacial erosion. Earth Surface Processes and Landforms, 22 : 297-306.

Linton, D.L., 1955. The problem of tors. Geographical Journal, $121: 470-487$.

Migon, P., 1997. Post-emergence modification of marine cliffs and associated shore platforms in a periglacial environment, SW Spitsbergen : Implications for the efficacy of cryoplanation processes. Quaternary Newsletter, 81 : 9-17.

Nesje, A. et Sulebak, J.R., 1994. Quantification of late Cenozoic erosion and denudation in the Sognefjord drainage basin, western Norway. Norsk Geografisk Tidsskrift $48:$ 85-92.

Rapp, A., 1996. Impact of mountain glaciations on tors, blockfields and cryoplanation features. Nunataks or non-scoured zones as refugia? In S.B. McCann et D.C. Ford, édit., Geomorphology Sans Frontières. John Wiley \&, Chichester, p. 137-152.

Ruddiman, W.F., 1977. Late-Quaternary deposition of ice-rafted sands in the subpolar North-Atlantic (lat. $40^{\circ}$ to $65^{\circ} \mathrm{N}$ ). Geological Society of America Bulletin, 88 : 1813-1827.

Söderman, G., Kejonen, A. et Kujansuu, R., 1983. The riddle of the tors at Lauhavuori, western Finland. Fennia, $161: 91-144$

Sugden, D.E., 1968. The selectivity of glacial erosion in the Cairngorm Mountains, Scotland. Transactions of the Institute of British Geographers, $45: 79-92$

1974. Landscapes of glacial erosion in Greenland and their relationship to ice, topographic and bedrock conditions. In E.H. Brown et R.S. Waters, édit., Progress in Geomorphology — Papers in Honour of David L. Linton. Institute of British Geographers, Special Publication 7 : 177-195.

1978. Glacial erosion by the Laurentide Ice Sheet. Journal of Glaciology, $20: 367-391$.

Sugden, D.E. et John, B.S., 1976. Glaciers and Landscape. Edward Arnold, London, $376 \mathrm{p}$.

Sugden, D.E. et Watts, S.H., 1977. Tors, felsenmeers and glaciation in northern Cumberland Peninsula, Baffin Island. Canadian Journal of Earth Sciences, $14: 2817-2823$

Watts, S.H., 1983. Weathering processes and products under arid arctic conditions (a study from Ellesmere Island, Canada). Geografiska Annaler, 65A : 85-98. 